\title{
Noncutaneous Melanoma
}

\author{
Jeffrey F. Scott \\ Meg R. Gerstenblith \\ Editors
}

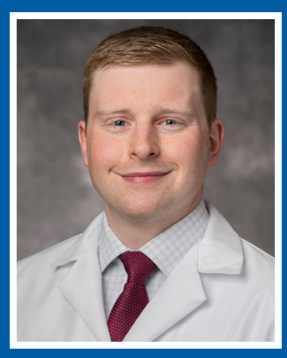

Jeffrey F. Scott, MD is a fellow in Micrographic Surgery and Dermatologic Oncology at University Hospitals Cleveland Medical Center and a clinical assistant professor in the Department of Dermatology at Case Western Reserve University. He completed his dermatology residency at University Hospitals Cleveland Medical Center. Dr. Scott's clinical and research interests include melanoma epidemiology, high-risk skin cancers, and Mohs micrographic surgery.

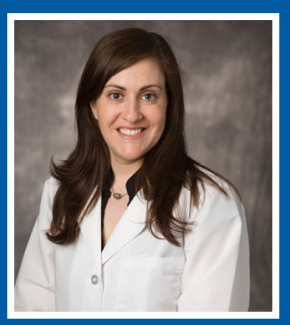

Meg R. Gerstenblith, MD is a clinical assistant professor in the Department of Dermatology at Case Western Reserve University. She completed her dermatology residency at Johns Hopkins Hospital, and completed a fellowship at the National Cancer Institute, National Institutes of Health, Division of Cancer Epidemiology and Genetics. Dr. Gerstenblith is a fellow of the American Academy of Dermatology, and her clinical and research interests include melanoma and pigmented lesions and the genetic epidemiology of skin cancer.

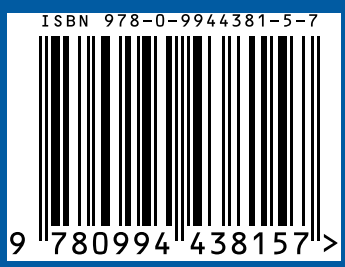

